\title{
Foreign bodies in the nasal cavities: a comprehensive review of the aetiology, diagnostic pointers, and therapeutic measures
}

\author{
A Kalan, M Tariq
}

\begin{abstract}
Otolaryngologists frequently encounter nasal foreign bodies, particularly among children and mentally retarded patients. There are isolated reports describing the removal of unusual foreign bodies from the nose. But no comprehensive reviews of this important subject have been published for many years. This article includes a detailed discussion of the different types of nasal foreign bodies, the various clinical presentations, management options, and complications associated with nasal foreign bodies.

(Postgrad Med F 2000;76:484-487)
\end{abstract}

Keywords: foreign bodies; nose

Several articles have been described in the otolaryngology literature describing unusual foreign bodies removed from the nasal space and the nasopharynx. There have been no comprehensive discussions on the subject of foreign bodies in the nose. Seldom does the otolaryngologist keep track of the number and kinds of foreign bodies he encounters. It is such a common entity that it is uncommon to pay that much attention to it. Foreign bodies in the nasal cavity, however, can be a great challenge and management may require great skill. ${ }^{1}$

At the very outset diagnosis could prove difficult. To the unsuspecting, a unilateral suppurative or mucopurulent fetid nasal discharge may suggest a number of possibilities other than a foreign body. On the other hand, the presence of a foreign body may be suspected but to prove its presence may be a trying task.

Department of Ear, Nose, and Throat Surgery, Royal Hospitals NHS Trust, London, UK A Kalan M Tariq

Correspondence to: Mr A Kalan, 136 Barrowell Green, Winchmore Hill, London N21 3AX, UK (email:

ak@kalan1.demon.co.uk)

Submitted 9 March 1999 Accpeted 27 September 1999

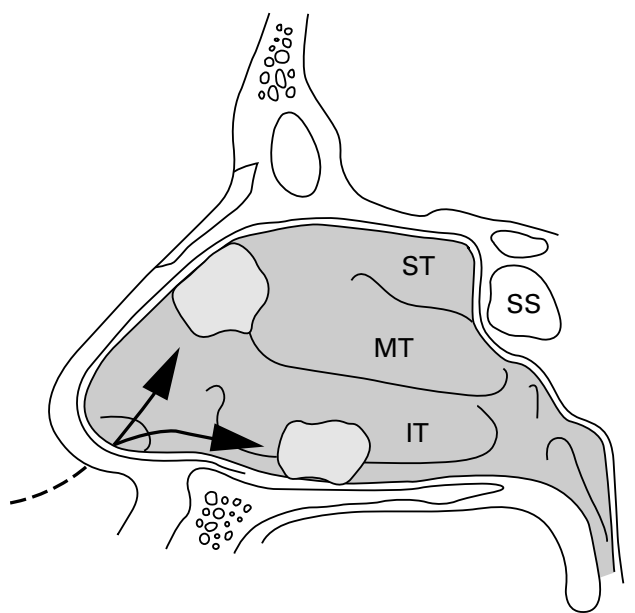

Figure 1 Common sites of impaction of foreign bodies in the nasal cavity (IT = inferior turbinate; $M T=$ middle turbinate; $S S=$ sphenoid sinus; $S T=$ superior turbinate).
A high index of suspicion is necessary so that further diagnostic manoeuvres can be tried before a label of "no foreign body" is stamped on the case.

Nasal foreign bodies can be found in any portion of the nasal cavity, although they are typically discovered around the floor of the nose just below the inferior turbinate (fig 1). Another common location is immediately anterior to the middle turbinate. ${ }^{2}$ These objects are generally placed by children or mentally retarded patients whose curiosity leads them to explore body orifices. Any article small enough to be admitted into the anterior nasal orifice has, at one time or the other, been removed from the nose. ${ }^{13}$ Foreign bodies that are impacted or those that have been present for some time and have become encrusted or those that have been impacted with force frequently challenge removal. Live and mobile animate foreign bodies (such as maggots and larvae) pose similar difficulties.

A loose foreign object in the postnasal space can accidentally be aspirated or pushed back in an attempt at removal and may result in acute respiratory obstruction. Foreign bodies in the nose have been implicated as carriers of the causative organisms of diphtheria and other infectious diseases. ${ }^{4}$ It therefore appears that foreign bodies in the nose can create a real problem and should not be taken lightly.

Foreign bodies are either animate or inanimate.

Inanimate foreign bodies - The list of objects that have been reportedly removed from the nose is endless. The most commonly identified inanimate foreign bodies include rubber erasers, paper wads, pebbles, beads, marbles, beans, safety pins, washers, nuts, sponges, and chalk. ${ }^{25}$ Other authors have reported plasticine, pieces of wood, a door handle, ${ }^{6}$ metal hooks and eyes, pieces of cloth, bullets, thimbles, ${ }^{7}$ shrapnel, umbrella springs, iron bolts, corks, and coins. ${ }^{8}$

Endogenous materials like bone and pieces of cartilage have been left behind in the nasal cavity after surgical intranasal manipulations. Trauma to structures adjacent to the nose such as orbits, paranasal sinuses, and palate can force bone spicules and cartilage fragments into the nose. Supernumerary teeth have erupted in the floor of the nose, presented like osteoma, and caused nasal obstruction..$^{9} 10$

Animate foreign bodies-Myiasis of the nose is common in warm tropical climates of South Western United States and the Far East including India, the frequency of infestation being primarily related to the poor hygiene of 
the inhabitants. The most common of all infestations is the fly maggot. Screw worms, also known as "Texas" screw worms (which is the larval state of Cochliomya macellaria and the Cochliomyia homnivorax, "the blow fly") are serious pests to humans and cattle. A high incidence of human infestation would therefore be expected in cattle raising areas but this does not appear to be the case. ${ }^{11}$ This indicates that the principal factor in infestation is not the livestock but rather the constitution and habitat of the host. The ordinary maggot represents the larval stage of this blow fly. It thrives in dead tissue only and does not destroy living material. The mature larva measures two thirds of an inch long and one eighth of an inch in diameter and has the characteristic appearance of a screw because of approximately 12 rings of spines that encircle the body. The larva burrows into the living tissue and it is at this stage of larval development that it damages nasal mucosal lining. Larvae of other flies like those of aestrous, hypoderma, and dermatobia also invade the nasal cavities. Wohlfahrtia magnifica may also infest the nose. These infestations occur more commonly in patients suffering from ozaena and nasal syphilis. ${ }^{1}$

Ascaris lumbricoides is a species of nematode or round intestinal worms and will find lodgement in the nose when regurgitated or coughed up. It is the most common intestinal helminth of man and frequently reaches epidemic proportions. Although it flourishes best in warm, tropical climates, it is cosmopolitan in distribution. In temperate regions it is generally associated with low standards of personal hygiene.

\section{Pathology}

Some foreign bodies are inert and may remain in the nose for years without mucosal changes. However, most inanimate objects initiate congestion and swelling of the nasal mucosa, with the possibility of pressure necrosis producing ulceration, mucosal erosion, and epistaxis. The retained secretion, the decomposed foreign body, and the accompanying ulceration can result in foul fetor. These changes further impact the foreign body because of surrounding oedema, granulations, and discharge. This is particularly seen with vegetable foreign bodies which not only absorb water from the tissues and swell but also evoke a very brisk inflammatory reaction. Occasionally, the inflammatory reaction is sufficient to produce toxaemia.

A foreign body can act as a nucleus for concretion if it is firmly impacted or is buried in granulation tissue by receiving a coating of calcium, magnesium phosphate, and carbonate and thus becomes a rhinolith. Occasionally this process may occur around an area of inspissated mucopus, or even a blood clot. Rhinoliths usually form near the floor of the nose and are radio-opaque.

Button batteries may result in severe destruction of the nasal septum. ${ }^{12}$ These are composed of various types of heavy metals: mercury, zinc, silver, nickel, cadmium, and lithium. Liberation of these substances causes

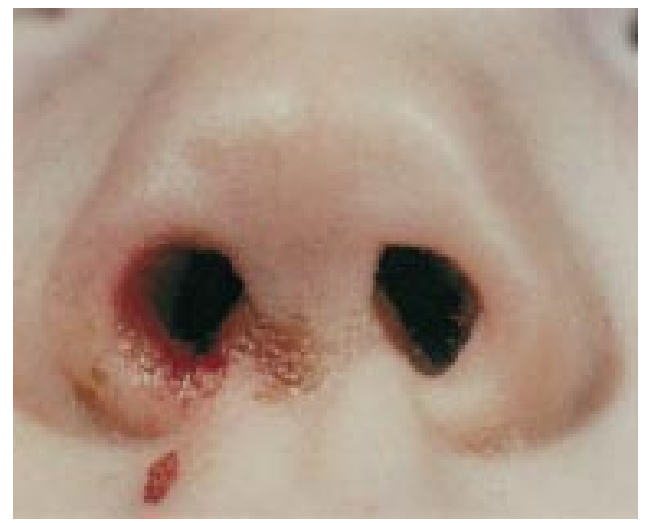

Figure 2 Unilateral vestibulitis in a child-diagnostic of a foreign body in the nasal cavity.

various types of lesions depending on the localisation, with an intense local tissue reaction and liquefaction necrosis. As a result they can cause septal perforations, synechiae, constriction, and stenosis of the nasal cavity.

Maggots and screw worms in the nose initiate varying degrees of inflammatory reaction from a mild localised infection to maximum destruction of the nasal bones (both cartilaginous and bony) with formation of deep, stinking suppurating caverns. The larvae hatch in these caverns and a new cycle is repeated. ${ }^{11}$

\section{Symptoms and signs}

A unilateral mucopurulent nasal discharge with foul odour is the most consistent findings in patients with a nasal foreign body. Occasionally it can be bloodstained. ${ }^{13}$ The ensuing unilateral vestibulitis, specific of the paediatric age group, is diagnostic (fig 2).

Nasal foreign bodies are generally painless. In fact some foreign bodies have reportedly been present in the nasal cavity for years without symptoms. ${ }^{14}$ However rarely, pain and headache have been experienced on the involved side with intermittent epistaxis and sneezing reported by others. ${ }^{13}$ Cases describing bromhidrosis (foul body odour) associated with nasal foreign bodies in children have also been published. ${ }^{15}$

In patients with animate nasal foreign bodies, the symptoms tend to be bilateral. ${ }^{113}$ Nasal occlusion, headaches, and sneezing with serosanguinous discharge usually are the presenting symptoms. A rise in body temperature occurs and a disagreeable fetid odour emanates from the nasal passages. Leucocytosis results from the accompanying secondary infection. Examination of the nasal cavity may reveal extensive destruction of the surrounding mucous membranes, bone, and cartilage and the mucosa is fragile and bleeds easily. Constant motion and masses of different worms may be observed. These worms are firmly attached and difficult to extract. Owing to the secondary infection and bone destruction, complications are not infrequent. ${ }^{13}$

Rhinoliths are initially symptomless and later cause nasal obstruction only if they become enlarged. Examination of the nasal cavity shows a greyish irregular mass, usually along the floor of the nose that feels bony, hard, and 
gritty on probing. Radiography usually confirms the diagnosis and reveals the extent of the rhinolith.

Any patient who presents with a unilateral nasal discharge should raise the suspicion of a nasal foreign body and in children this must be regarded the case until proved otherwise. The physical examination of the nose involving anterior rhinoscopy and use of either a fibreoptic nasopharyngoscope or a 0 degree rigid endoscope will often reveal the foreign object. However on occasions mucosal oedema or granulations tend to hide it. In such cases the nose should be sprayed with a vasoconstrictor agent to shrink the mucosa before reexamination. Many times the foreign body becomes apparent with this manoeuvre. ${ }^{13}$ In younger or very apprehensive children it may be necessary for the search to be carried out under a general anaesthetic.

In patients with parasitic and larvae infestation of the nose diagnosis is relatively easy as the organisms are directly visualised.

Several unilateral lesions found in both children and adults may produce obstruction of the involved side. Such lesions would include both benign and malignant tumours of the nasal cavity, unilateral sinusitis, unilateral choanal atresia, unilateral nasal polyps, septal haematoma, and infections like syphilis and diphtheria. ${ }^{16}$

\section{Management}

A cooperative patient is needed to detect and remove a nasal foreign body successfully. The patient is usually examined in the upright sitting position carried out for routine otorhinological examination. A child may be best examined by tilting the head back slightly so that the floor of the nose is visible to the examiner. For this an adult may need to restrain a child and hold the head steady.

Most inanimate foreign bodies, if visualised well, can be removed easily through the anterior nares with the use of cupped forceps, haemostats, curved hooks, old metallic eustachian tube catheters, and suction. This can be done either with no anaesthetic or after spraying with a local topically acting anaesthetic solution such as $4 \%$ lignocaine (lidocaine). Removal of a rounded object may be an arduous task because of difficulty in grasping foreign bodies of this shape. A curved hook is best suited for this job. The hook is first passed behind the object, the tip rotated to rest just behind it and then the foreign body is gradually drawn forwards and out through the nose. ${ }^{17}$ Additionally, several suction methods have been described that aid in the removal of round foreign bodies. ${ }^{18}{ }^{19}$ Plastic objects and vegetable matter may be difficult to extract because of their tendency to break into small pieces.

However, it cannot be too strongly emphasised that unskilled attempts to remove the foreign body in accident and emergency departments by a person without appropriate training may result in disaster. The foreign body may be displaced backwards and may even reach the nasopharynx with a risk of inhalation. ${ }^{13}$ In a crying child the foreign body while being

\section{Learning points}

Several points need to be emphasised when dealing with nasal foreign bodies:

- Nasal foreign bodies are commonly encountered in emergency departments, particularly among children and mentally retarded patients.

- The diagnosis should be entertained in any patient who presents with a persistent unilateral nasal discharge.

- Nasal foreign bodies are either inanimate objects or, less commonly, animate.

- Successful diagnosis and treatment of nasal foreign bodies depends on careful examination of the nasal cavity and its adjacent structures.

- Medical personnel skilled in the technique of removal of nasal foreign bodies should be involved from the outset.

- Due to lack of cooperation from the patients and occasional difficulty in extraction of nasal foreign bodies general anaesthesia should be considered if there is any question arising concerning the adequacy of nasal examination.

removed from the nose can fall into the mouth with calamitous effects. Marked epistaxis may occur or a docile child may become terrified and require a general anaesthetic, which might otherwise have been avoided. ${ }^{13}$

In cases in which removal of a foreign body is particularly difficult, several alternative procedures have been described. If the patient can cooperate, they can be instructed to take a deep breath through the mouth and then forcibly exhale through the nose. The attending doctor should occlude the uninvolved nostril during this procedure. ${ }^{20}$ If the patient is not able to cooperative with this manoeuvre, forced mouth-to-mouth ventilation can be administered to the patient by the doctor, again occluding the uninvolved side. ${ }^{21}$ Both the success rate and the incidence of complications associated with the above mentioned procedures are not well reported in the literature.

An additional method described by some authors is the use either of a Foley catheter or a Fogarty biliary catheter for removal of nasal foreign bodies. ${ }^{22} 23$ After ensuring that the balloon is intact, the catheter is passed into the nose beyond the foreign body. The balloon is then inflated with $0.5 \mathrm{ml}$ of water. The catheter is withdrawn back through the nose, pulling the foreign body in front of the balloon.

In rare cases, the only successful method of removing a nasal foreign body is to push the object posteriorly into the pharynx. ${ }^{24}$ In these cases a general anaesthetic is required and endotracheal intubation performed to protect the airway.

Different techniques are employed for removal of animate foreign bodies. In the case of screw worms, larvae and maggots, a weak solution of $25 \%$ chloroform is instilled into the nasal spaces to kill the larvae. This may have to be repeated two or three times a week for about six weeks until all larvae are killed. After each 
treatment, removal can be accomplished by blowing the nose if the patient is awake and by suction, irrigation, or curettage if the patient is asleep.

With Ascaris lumbricoides manual or forceps extraction is utilised thus avoiding the need for killing these worms before removal. The roundworms that become lodged in the nose, however, present only a part of the heavy intestinal infestation that has to be eradicated in order to prevent further nasal lodgement. Oral levamisole or mebendazole may be used for this.

After successful removal of a nasal foreign body, careful examination of the involved nasal cavity as well as the other body orifices must be undertaken to exclude the presence of other unrecognised foreign bodies. Particular attention must be paid to the examination of the ear and sinuses on the involved side as acute otitis media or sinusitis are commonly seen if the foreign body has been present for any length of time. Additionally, expistaxis which frequently accompanies the removal of nasal foreign bodies must be appropriately dealt with.

Several important complications have been associated with the presence of a nasal foreign body. These include formation and development of rhinoliths, erosion into a contiguous structure, and producing infections in surrounding structures. Apart from acute sinusitis or otitis media, other infections reported include periorbital cellulitis, meningitis, acute epiglottitis, diphtheria, and tetanus. ${ }^{25} 26$

The authors would like to thank Mr G S Kenyon, Consultan Ear, Nose, and Throat Surgeon for his guidance in writing this manuscript and Mrs Ross Doyle for secretarial services. 1 Baluyot ST. Foreign bodies in the nasal cavity. In: Paparella
MM, Shumrick DA, eds. Otolaryngology. Philadelphia, WB Saunders, 1980: 2009-16.
2 DeWeese DD, Saunders AH. Acute and chronic diseases of the nose. In: DeWeese DD, Saunders AH, eds. Textbook of otolaryngology. St Louis: CV Mosby, 1982.

3 Kelemen G. Spontaneous expulsion of a foreign body by transmigration through the nasal wall after 28 years. Laryngoscope 1955;55:375-9.

4 Burton AH, Balmain AR. Foreign bodies and nasal carriers of diphtheria. Lancet 1929;ii:977.

5 Guthrie D. Foreign bodies in the nose. 7 Laryngol Otol 1956;41:454-7.

6 Malhotra C, Arora MM, Mehra YN. An unusual foreign body in the nose. 7 Laryngol Otol 1930;45:73.

7 T'Ang CY. A large foreign body in the nasopharynx of an infant. F Laryngol Otol 1954;68:321-3.

8 Irvine GS. A case of foreign body in the nasopharynx. $f$ Laryngol Otol 1960;74:188-91.

9 Hiranandani LH, Melgiri RD. Supernumerary tooth in the floor of the nose. F Laryngol Otol 1968;82:845-8.

10 Kohli GD, Verma PL. Ectopic supernumerary tooth in the nasal cavity. F Laryngol Otol 1970;84:537-8.

11 Brown E. Screw worm infestation in the nasal passages and paranasal sinuses. Laryngoscope 1945;55:371-4.

12 McRae D, Premachandra DJ, Gatland DJ. Button batteries in the ear, nose and cervical oesophagus, a destructive foreign body. F Otolaryngol 1989;18:317-19.

13 Walby AP. Foreign bodies in the ear or nose. In: Kerr AG, Walby AP. Foreign bodies in the ear or nose. In: Kerr AG,
ed. Scott-Brown's otolaryngology. 6th Ed. Oxford: Butterworth-Heinemann, 1997: 6/14/1-6/14/6.

14 Goldsten E, Gottlieb MA. Foreign bodies in the nasal fossae of children. Oral Surg Oral Med Oral Pathol 1973;36:446-7.

15 Golding IM. An unusual cause of bromhidrosis. Pediatrics 1965;36:791-2.

16 Myer CM, Cotton RT. Nasal obstruction in the paediatric patient. Pediatrics 1983;72:766-77.

17 McMaster WC. Removal of foreign body from the nose. fAMA 1970;213:1905.

18 Morris MS. New device for foreign body removal. Laryngoscope 1984;94:980.

19 Jensen JH. Technique for removing a spherical foreign body from the nose or ear. Ear Nose Throat f 1976;55:270-1.

20 Messervy M. Forced expiration in the treatment of nasal foreign bodies. The Practitioner 1973;210:242.

21 Stool SE, McConnel CS. Foreign bodies in paediatric otolaryngology: some diagnostic and therapeutic pointers. Clin Pediatr (Phila) 1973;12:113-16.

22 Fox JR. Fogarty catheter removal of nasal foreign bodies. Am Emerg Med 1980;9:37-8.

23 Henry LN, Chamberlain JW. Removal of foreign bodies from oesophagus and nose with the use of a Foley catheter. Surgery 1972;71:918-21.

24 Harner SG. Foreign bodies in the ear, nose and throat. Postgrad Med F 1975;57:82-3.

25 Oh TH, Gaudet T. Acute epiglottitis associated with nasal foreign body: occurrence in a 30 month old girl. Clin Pediatr (Phila) 1977;16:1067-8.

26 Sarniak AP, Venkat G. Cephalic tetanus as a complication of nasal foreign body. Am $\mathcal{F}$ Dis Child 1981;135:571-2 